Voix et Images

volxetimages

\title{
Emmanuel Cocke, l'amphigouri ou les esquives de la stupeur
}

\section{Élène Cliche}

Volume 9, numéro 3, printemps 1984

Monique Bosco

URI : https://id.erudit.org/iderudit/200481ar

DOI : https://doi.org/10.7202/200481ar

Aller au sommaire du numéro

\section{Éditeur(s)}

Université du Québec à Montréal

\section{ISSN}

0318-9201 (imprimé)

1705-933X (numérique)

Découvrir la revue

\section{Citer cet article}

Cliche, É. (1984). Emmanuel Cocke, l'amphigouri ou les esquives de la stupeur.

Voix et Images, 9(3), 85-101. https://doi.org/10.7202/200481ar d'utilisation que vous pouvez consulter en ligne.

https://apropos.erudit.org/fr/usagers/politique-dutilisation/ 


\title{
Emmanuel Cocke, l'amphigouri ou les esquives de la stupeur
}

\author{
par Elène Cliche, Université du Québec à Montréal
}

\begin{abstract}
Tentation de l'écrivain
Avoir écrit quelque chose qui te laisse comme un fusil qui vient de tirer, encore ébranlé et brolant, vidé de tout toi, ou non seulement tu as déchargé tout ce que tu sais de toi-même mais ce que tu soupçonnes et supposes, et les sursauts, les fantômes, l'inconscient - avoir fait cela au prix d'une longue fatigue et d'une longue tension, avec une prudence faite de jours, de tremblements, de brusques découvertes et d'échecs, et en fixant toute sa vie sur ce point - s'apercevoir que tout cela est comme rien si un signe humain, un mot, une présence ne l'accueille pas, ne le réchauffe pas - et mourir de froid - parler dans le désert - être seul nuit et jour comme un mort.
\end{abstract}

(extrait du journal le Métier de vivre) ${ }^{1}$

Cesare Pavese, 27 juin 1946

Fidèle à sa légende suicidaire, Emmanuel Cocke s'est noyé dans les eaux indiennes de Pondichéry, en septembre $1973^{2}$. Immortel sans contredit, selon son vou réitéré et sa volonté ferme de revenir. («Alors il s'aperçut qu'il avait été victime d'un parasitage, et en faisant son bilan personnel, il s'aperçut qu'il était mort prématurément, que ses références pour passer de l'autre côté du chronomètre n'étaient pas assez sérieuses, et qu'il devait retomber sur terre.» Préface à Sexe-fiction). Ainsi se présente dix ans après le spectre de son œuvre méconnue dont l'imbroglio de signes se donne à voir-entendre désormais sur une autre scène, un autre espacetemps, interrogeant par conséquent notre désir actuel d'inspecter et d'arpenter des lieux désertés et abandonnés. L'écho ne répercutera-t-il que notre propre voix?

D'abord, rappelons que le corpus publié de l'auteur comprend quatre romans : Va voir au ciel si j'y suis (Mtl, Éd. du Jour, 1971), l'Emmanuscrit de la mère morte (Mtl, Éd. du Jour, 1972), Louve storée (Mtl, Vert blanc rouge/Éd.de l'Heure, 1973), Sexe pour sang (Mtl, Éd. Guérin, 1974), et un recueil de nouvelles : Sexe-fiction (Mtl, Éd. de l'Heure, 1973), contenant l'adaptation de deux pièces radiophoniques, d'une nouvelle radiophonique, d'un texte conçu pour un projet de bande dessinée, etc. Il existe par ailleurs de nombreux textes inédits ${ }^{3}$. 
Nous oserons affirmer que chez Emmanuel Cocke, l'ardent vouloirécrire, le désir brûlant de produire une œeuvre et de se construire une légende, enfin le processus même d'engendrement est beaucoup plus important et séduisant que ce qui en résulte, les livres produits. Ainsi, au lieu même du surgissement de la parole, l'œuvre tire ses conséquences ultimes : à savoir qu'il n'y a pas d'autre vérité que ce qu'un sujet désire. Les pulsions étant les forces du désir, le rapport du sujet au langage sera donc essentiellement pulsionnel, et ainsi le sujet de l'écriture qui nous occupe, ce «mirliflore mirifique ${ }^{4}$ » affichera sa passion ludique du signifiant, et cela sans complaisance pour la lecture. Les fluctuations de cette alacrité incluront nécessairement et grossièrement les ratages et retombées médiocres du mouvement, constituant par conséquent une esthétique du discours amphigourique, compréhensible et incompréhensible, délibérément brouillon, sauvage, cru, discontinu, à vitesses différentes. "Tâchez d'être son aboulique aficionado» (VVC p. 138).

La littérature mineure (Deleuze), celle qu'une minorité fait dans une langue majeure, les agencements d'énonciation comme déterritorialisation de la langue nous invitent à penser l'écriture en terme d'effets, et ici en l'occurrence, effets de dispersion sous l'action du morcellement. Ainsi, le mode de lecture est davantage réglé par une économie de forces, une distribution des unités d'énergie selon lesquelles s'effectue l'alternance pulsionnelle : cybernétique, mysticisme, drogue, sexe, sang, apocalypse, etc. Le discours amphigourique, auquel se rallient plusieurs textes de la modernité, nous amène à établir, du moins si l'on veut bien faire vibrer les séquences sur des intensités, des inventaires de signes discontinus sur le plan paradigmatique, à reconstituer des lexiques, des réseaux combinatoires, bref une systématique de marques corrélatives, entre autres des séries nominales abolissant leur divergence dans le jeu de la différence. (Ceci afin de montrer le "caractère différentiel" du fonctionnement sémiologique, soumis aux especes de la contradiction et de la logique multiple).

Dans une étude antérieure plus détaillée sur la pratique textuelle d'Emmanuel Cocke (mémoire inédit), nous avions établi comme hypothèse opérationnelle, envisageant l'énergie cinétique de la textualité, un diagramme sous forme de ligne asymptotique, celle-ci pointillée par des unités signifiantes en relation de disjonction alternante, à partir de quoi venaient se brancher d'autres unités ouvrant des régimes de signes différents, en offrant de multiples possibilités de circuit. Nous ne reprendrons pas cette typologie mouvante d'éléments signifiants et les traits qui s'y rattachent dans le cadre restreint de cet article. L'analyse plus approfondie du discours permettait de réactiver le tissu des signes en mettant ceux-ci en rapport de recoupement, de glissement ou d'éloignement. Actuellement, il nous importe peu d'établir des organisations destinées à restratifier l'ensemble de l'oeuvre vers une totalité signifiante (malgré le désir du total chez Cocke, style total, monde total, «Sois totalibre!», SF p. 22), alors que les ruptures du texte montrent 
davantage une déstratification. renouvelée. Nous voulons seulement nommer un désir - son sujet - c'est-à-dire l'émergence dispersante qui contribue à la fabrication du texte cockien et déterminant l'esthétique qui $s$ 'en dégage. Le «malheur» de cette articulation sera de constater en définitive, et cela contre notre gré, que les effets dispersants sont les maquillages de ce qui n'arrive pas à se disperser, que l'on nomme cela la Dispersion même, le Réel, l'horreur - je dis la stupeur.

Il est éminemment facile de ramener l'amphigouri en tant que réalité hétérogène de la langue à une scène fondamentale, à une interprétation téléologique, unaire, à la réduction des lois de combinaison, à la prise de la multiplicité dans la structure de l'Un. Le texte de Cocke s'y prête fort bien, c'est ce que nous appellerons plus loin le point $\mathrm{mmm}$, ce centre d'attraction toujours prêt à exploser.

Mais la signifiance comme production du sens à partir des signifiants est l'entreprise destinée à décentrer sans cesse le langage sur plusieurs dimensions ${ }^{5}$. Nous rattachons arbitrairement ceci au réel dispersant, le réel entendu ici au sens lacanien et repris par Jean-Claude Milner dans les Noms indistincts ${ }^{6}$ comme agrégat où ne s'établit nul lien, nulle propriété, nulle similitude ou dissimilitude. C'est l'impossible, le moment de la dispersion étant le moment du déconcertant. Tel que l'indique Milner, les noms variés viendront disperser les Maîtres-mots, mais ce n'est que la menue monnaie du dispersant radical, soit le sujet et son désir en ce qu'ils ont de réel ${ }^{7}$. Nous pensons donc la dispersion comme un mode issu d'un geste de coupure qui éparpille et pulvérise, ses effets sont les travestissements du réel anéantissant, esquives de la stupeur grâce d̀ l'imaginaire qui crée le lien et au symbolique discriminateur. Ainsi la Mort dispersante entrelacée d'Eros est un principe de désorganisation du texte et du sujet désirant qui ne passe pas au sujet maîtrisé. De ce sujet ainsi délié, émerge chez Cocke l'acte d'écrire et les actes (d'énonciation) de déphasage produits dans la langue.

Les effets se marquent à plusieurs niveaux. Eclatement des genres et des tons : science-fiction, policier, pornographie, bande dessinée, autobiographie, récit d'aventures, fiction utopique, chanson, etc. Comme une chaîne de démons (SpS p. 85), l'identité se disperse de Jésus Tanné à Brokendov à Dieuble à Serge Marcus etc., de Calmoya à Mauve à Liz à Virago a Tupetu 1-2-3, Geneviève Thomas etc., ainsi que la signature en métamorphose dans les réseaux éclatés de l'onomastique textuelle. Vian, Cervantès, Baudelaire, Rimbaud, Nerval, Pavese, Burroughs, Mailer, Miller, Céline, Breton, Joyce, Unamuno, Kafka, Hesse, Péloquin, Swedenborg, etc. Dans le jeu de la différence patronymique chez Cocke, s'affirme le décharnement de l'individu imaginaire homogénéisant, le pasmoi de la signature. Je est dans la chaîne divine du démoniaque.

Dispersion du mot, la lettre permettant de nouveaux agencements, nouvelles matières de jeu. Ainsi en est-il des situations romanesques dans 
l'espace et dans le temps, histoires «coquecigruesques», plaisir de raconter des balivernes. Le Lien malgré tout est sauvé («le truc pour sauver ses semblables d'une Fin du Monde probable», VVC p. 206), mais il donne constamment à lire son suspens. Le ton est discontinu, la langue d'écriture de Cocke est hors-famille, elle intègre des modèles allogènes avec émergences éparses de joual, d'anglais, d'argot, mots d'origines diverses. Par exemple, le nom Brokendov condense «broken down» avec la syllabe "dov» à consonance russe. L'incorporation des modèles allogènes sera beaucoup plus poussée chez des poètes modernes français tels que Denis Roche $^{8}$; chez Cocke le geste demeure mitigé pour plusieurs raisons sur lesquelles nous n'allons pas élaborer, mais il est toujours décelable, relié à la peur-stupeur. La nouvelle «Les ennemis du joualbum» (SF p. 35-59) pose le problème de "lalangue» (Lacan désignant ainsi la langue orale, la chaîne des noms), le joualbum étant un mélange d'anglais, de français, d'espagnol, de portugais et d'autres dialectes, dont «ce ne sont pas tellement les mots qui comptent, mais les sons» (SF p. 35). Le joualbum imaginé comme langue officielle s'affronte au français, «cette langue décadente dont les mots, trop nombreux, s'entrechoquent comme des vieilles pierres, dès qu'on essaie de les articuler, et dont les syllabes dérapent sur les lèvres, à moins de les prononcer lentement» (SF p. 37-38).

Cocke est un verbalisateur et le langage est visé par la satire. Ça disperse. Le narrateur de l'Emmanuscrit se réclame du principe de la «clinamen», une idée "comme un gag», la déviation spontanée grâce à laquelle les atomes tombant dans le vide s'écartent de la ligne droite et peuvent ainsi se rencontrer et s'accrocher pour former les divers corps (EMM p. 110). Ce qui sera appelé la technicalité du Farfelu (LS p. 109) témoigne d'un mépris pour les ensembles dicibles, s'attaquant ainsi au fait même de dire. C'est le règne des bavures de langage, d'un signifiant toujours pris pour un autre, la dé-prise, bien que l'écriture soit également conformiste, soumise aux normes de l'expression et de la communication. Pas une véritable schizologie ${ }^{9}$. Simplement un geste qui fait subir à la loi et au code de la langue un travail scriptible producteur de jeux de mots ${ }^{10}$, presque mécanique chez Cocke grâce à une sensibilisation extrême au matériau. La langue devient un terrain d'expérimentation, le jeu de mots naïf et puéril met à l'épreuve les ressources illimitées du signifiant. «(...) chaque mot est un siège éjectable pour le regard» (EMM p. 49). De même qu'il n'y a pas de différence entre ce dont un livre parle et la manière dont il est fait (Deleuze-Guattari), la représentation d'un «monde renversé» dans Va voir au ciel si j'y suis exige que les mots le soient : inscription des lettres à rebours «Laertnom» «Ednom», «telliuj», «Cebeuq» etc. Altération de l'ordre des lettres, «hénoïre», "cacoïne», "phormine», «upiom» etc. La transformation est une règle du jeu, le texte est ouvert à l'extension des signifiants comme la série des Miss (VVC p. 93). L'espace substitutif homonymique du sujet désirant dans «lalangue», «passeporc» (VVC p. 24), «nez en moins» (VVC p. 86), «seins-bal» «seins-bol» (VVC p. 97) «-Toi ne prend pas de t. - Je ne prends pas de thé» (VVC p. 77), ce que le 
narrateur appelle «le ton dictaphonique» (VVC p. 52) produisant du nonsens, du sens nouveau, «sangsuelle» (VVC pp. 81, 119, 122) donc la mise en relief de cet espace est en ordre décroissant - chronologiquement - dans le corpus. Dans les derniers textes, l'homonymie, qui est le Réel de lalangue là où tout nom est multiplement ambigu, est pratiquement inexistante. Quelques traces «mort fine» (LS p. 9), «messes câlines» (LS p. 76), «as en soeur» (SF p. 63), mais le texte sera davantage prisonnier du signe.

Le travail sur le signifiant provoque de multiples distorsions anagrammatiques d̀ travers lesquelles l'énonciation se déguise et s'esquive dans les processus de déplacement et de condensation : «mégot centrique» (VVC p. 61, 122), "pantacourt» (VVC p. 102, LS p. 32), «Edam, Ave» (VVC p. 175), «Atlantigre» (EMM p. 119). Les exemples sont nombreux du possible à conquérir dans le champ du désir de la langue, de son espace producteur. Les jeux de mots pratiqués dans et par le texte répondent à l'envie de disperser, de défaire le fixé, détruire le standardisé. La forme toujours en devenir fait de l'espace textuel cockien un espace brisé, car l'activité ludique pratiquée opère des ruptures dans l'articulation d'une "compréhension» soutenue et continue du texte, «en ce sens insensé») (VVC p. 1) qu'elle pratique sans cesse des ouvertures dans le tissu. Le sens est décoloré dans la pratique matérialisée du texte, ceci contribue à protéger les sources génératrices de plaisir. Métaphore et métonymie servent à tromper et à simuler, c'est une ruse de l'écriture et une jouissance dans les frétillements de cette expérience de déboîtement du signe. L'apatride se trouve une patrie, les mots.

L'écriture d'Emmanuel Cocke est fortement conditionnée par des langages culturels (codes de savoir) formant des combinatoires signifiantes. Il faut tenir compte également du langage contre-culturel, daté historiquement, celui d'un circuit "underground» des années 65-70, auquel se rattachent les noms de Bob Dylan, Andy Warhol entre autres, le psychédélisme, les hippies, le LSD, etc. Le code littéraire est foisonnant, il fournit des alibis au texte de Cocke qui s'étabit comme l'enclave d'autres textes (Cf. note 2 sur C. Gauvreau). Par exemple, le Métier de vivre de C. Pavese (LS pp. 8, 119), ne serait-ce que ce titre, est un investissement constant dans l'œuvre de Cocke jusqu'à Auroville. La ligne de fuite «mais les suicidés ont du panache» (Gauvreau), rejoignant la ligne pavésienne de l'identification à la crise d'un sujet abandonné par une actrice, identification au sujet humilié, souffrant et paranoïde, est une des figures les plus fortes, sur laquelle le discours se connecte à maintes reprises parmi les divers agencements de projection du désir. Nous y reviendrons plus tard.

Les significtions se dispersent donc par une traversée de langages constituants, littéraire, cinématographique, musical, politique, économicosocial, scientifique - physique, chimique, médical - surtour dans Va voir au ciel si j'y suis, ce sont des éléments productifs dont l'acte pluriel de l'écriture va s'accaparer, tout en les dispersant. Nous n'allons pas déployer 
ici les taxinomies de ces diverses voix. Roland Barthes, à propos du texte de Bataille, relevait ces divers codes de savoir et montrait que le savoir s'en trouvait ainsi truqué par un émiettement des codes :

le roman est en effet une mathésis truquée, en route vers un détournement du savoir. Ce frottement de codes d'origines diverses, de styles divers est contraire à la monologie du savoir, qui consacre les «spécialistes» et dédaigne les polygraphes (les amateurs) ${ }^{11}$.

Dans le texte de Cocke, le savoir est hétéroclite et prétexte à un acte anthologique (disséminé) de nomination renouvelée dans différents champs : zoologie, ornithologie, botanique. Noms d'oiseaux, de reptiles, plantes, fleurs, etc., mêlés à une variation d'autres voix : message publicitaire, slogan, journalisme, chanson. Manifestation farfelue de divers codes, par exemple vestimentaire, «une chemise en écorce de bouleau plastifiée» (VVC p. 23), gastronomique, "une gaufre aux sangsues panées» (VVC p. 100), «des céréales d'orignal au miel et des coquelicots au jus de tibia des Montagnes Rocheuses» (EMM p. 92), etc., mystique, avec des figures éparses telles que Swedenborg, les Rose-Croix, Unamuno, Aurobindo, etc. Le texte construit un code gnomique, les maximes de sagesse sont reproduites comme des clichés : «la sensualité est supérieure à l'érotisme, tous les pornographes vous le diront» (LS p. 51); «Plus on reste rattaché aux choses, moins on reste intègre» (VVC p. 21); "L'action est la force des faibles» (VVC p. 50). Reconstituer le texte gnomique, la parole morale, étouffe la lecture. C'est un lieu où l'instance de l'écriture perd son pouvoir déformateur, on retrouve le signifié plat d'une espace monologique qu'il faut s'empresser de désarticuler. Le code cinématographique est pris comme modèle de création audiovisuelle. Cocke se disait un cinéaste frustré et voulait rentabiliser son désir en filmécrivant. Ceci renvoie à une forme de discours hallucinatoire, imaginons un texte-peyotl soumis au principe du rêve en tant que tissu d'impressions sensorielles, le plus souvent visuelles. Ceci nous ramène également au principe du montage et du démontage des signes.

Il existe un autre code qui s'entremêle aux autres dans la dispersion des lignes de sens, c'est le code misogyne. Le texte met en action une fantasmagorie phallique sous-tendue par une peur de la castration. L'amphigouri se nourrit de gags orgiaques, le burlesque sexuel s'articule au dévoyé : transes, danses, permutations sexuelles - hétérosexuelles - se branchant aux délires mégalomanes des héros mâles tels que Jésus Tanné ou Dieuble, ou à des fantasmes d'éviration. Dieuble : «Je suis un fou qui est folle» (EMM p. 43), condensation androgyne; dans Louve storée, être Geneviève Thomas, être l'autre, être femme. «Je respire mieux, mes seins se gonflent selon la courbe angélique du Bateau de l'Espoir» (LS p. 30) ${ }^{12}$.

Plusieurs énoncés sont branchés sur la ligne misogyne, proférés avec plus ou moins d'ironie : "d'une subtilité étonnante pour une femelle» (EMM p. 34). Les figures féminines classiques sont représentées sur le paradigme de la «Marie-Vierge» «naïve», «neuve», «tendre biche», 
«péronnelle», "petite sotte» (VVC pp. 20, 49, 81) et de la "Marie-salope», «slips et clips» (VVC pp. 15, 36). C'est davantage sur cette dernière figure que le texte va jouer et représenter la stratification sadique. Filles, femelles, putes, garces, nanas, «biches abîmées consentantes à la Débauche» (VVC p. 64), "corps putanesques pour petits dieux terrestres» (VVC p. 65), prêts à être dominés violemment. Série des verbes de la maîtrise, «Je la jette sans préavis sur ma couche divine» (VVC p. 16), «je catafalque», «je les talonne au ventre» (VVC p. 67), "Autant la prendre tant qu'elle est là» (VVC p. 76), «tandis que je culbute allègrement la sédentaire» (VVC p. 104), «de rendre mes femmes dégueulasses, de les vendre au dernier venu, pour finalement les balancer à la poubelle du Temps» (VVC p. 159), «je l'ai ranimée comme une belle au bois dormant» (EMM p. 149). Être Maître du sexe, autour de ce désir les signes s'amoncellent, et une équivalence fantasmatique s'établit entre baiser et signer son ceuvre (VVC p. 16). Cela s'articule à partir de la castration, de la faucille redoutée. Paradoxalement, Jésus Tanné se retrouvera contrôleur du châtrage généralisé dans le Système Ibéhème (VVC p. 70-71) dirigé par le Membre Viril (jeux de phallus). Devant les femmes à sodomiser, à tuer dans certains cas (VVC p. 151), ces "clownesses d'amour» telles que Tupetu «vicelarde, dézinguée, hystérique» (VVC . 66), le héros promu vedette, le seul Dieu (VVC p. 147) mégalomane est secrètement menacé. Ainsi émerge l'ambivalence de cette matière femme-pute-salope, que le texte investit des affects de la stupeur, de l'effroi anéantissant. Elle tue. Un processus déplace les termes du paradigme (Marie-Vierge, Marie-salope) vers un troisième terme plus insaisissable, c'est Marie Morgane, «née de la mer par nuit de pleine lune» (LS p. 10), figure de contradiction, de rêve, c'est la belle et perverse fée. Figure absorbante et anéantissante pour le sujet, provoquant une mise en éveil de la parole fantasmatique reliée à la mère phallique et archaïque, au mauvais sein. L'irruption de cette figure comme fantôme de désir - menaçant - va circuler dans toute l'ouure de Cocke. Du point de vue de l'énonciation, c'est le pôle d'interlocution par excellence, l'Interlocutrice Idéale du sujet parlant : Calmoya.

Calmo, place-moi sur un plasma nouveau, à quatre pattes sur les étoiles, l'esprit califourchonnant les vieilles planètes, l'âme tanguant sur le cosmos, je surchauffe (VVC p. 51).

C'est une figure d'identification, fonctionnant comme le double indispensable à l'image du sujet. La réflexion dans le miroir introduit un second narcissisme ${ }^{13}$ dont le "pattern» n'est plus simplement l'image corporelle, mais la relation à l'autre, l'alter ego, l'idéal du moi. Cette identification à l'autre, selon Lacan, permet au sujet de situer avec précision son rapport imaginaire et libidinal au monde en général. C'est ce qu'il appellera son être libidinal. Ceci est également déterminant dans l'équivalence qui sera faite entre cet idéal du moi et l'objet aimé, l'investissement amoureux par la captation que cet objet va opérer, la dépendance, «véritable perversion de la réalité par la fascination sur l'objet aimé et sa surestimation» ${ }^{14}$. Le signifiant Calmoya («c'est un nom tranquille et rassurant; ça remplace une mère, 
ce nom», VVC p. 13), dans lequel on peut entendre "Calme-moi», est un mot plein, par lequel le héros Jésus Tanné est électrifié, brûlé, anéanti, «sans elle je suis exfolié» (VVC p. 50). Lorsque cette figure émerge, comme de la mer, dans le texte, celui-ci dévie. Figure d'identification narcissique, «frère-soeur siamois» (VVC p. 51), de pouvoir, celui de la mise à mort. (C'est elle qui propose de détruire le ciel automatique et de balancer Jésus Tanné dans le vide (VVC p. 199), ce qui s'avèrera n'être qu'un simulacre, un leurre (VVC p. 203). Dans l'Emmanuscrit, ce fantôme flottant sur la mer rejaillit sous l'image de l'anémone de mer qui tue, accusant l'homonymie mer-mère, avec incidences autobiographiques ici; poison, venin, en conjonction avec la culpabilité de la réverbération narcissique, ce qui me tue, je l'ai tué(e). Cette figure féminine est matérialisée en actrice, histrionne insaisissable, persécutrice. Par la blessure narcissique qu'elle a fait subir au sujet (ce creuset pavésien), elle est portée garante de la castration, ajoutons également castration du logos en tant que ratio. Vampire, sorcière, déesse diablesse, absolument pute, «maudite femelle», être de métamorphose inaccessible, c'est Geneviève Thomas mère, soeur, fille, amante perverse, etc. (LS p. 47), que le protagoniste Alun Meexomec (anagramme de Emmanuel Coxe) adore et insulte. Nous savons que l'image de la femme porteuse de mort traverse plusieurs textes littéraires ${ }^{15}$. Ici, le sujet est prisonnier de l'image, l'écriture la constituant comme son «être libidinal» narcissique. Pas de dégagement explosif comme dans le texte de Sollers mentionné, bien que chez ce dernier l'on pourrait également prouver le contraire. Ici, le texte s'accroche à son double comme un «morpion affamé». Dans l'échec de la distanciation, il y a contrainte de répétition. "Aimer une morte qu'on a tuée et que l'on tue et retue chaque jour par orgueil, c'est fatigant, autant y passer soi-même» (EMM p. 112). La nouvelle Sexe-fiction est la mise en scène même de la castration, la représentation du se faire bouffer, attaquer et violer par le sexe féminin. Retournement violent des fantasmes de domination mâle déjà exposés.

Ceci nous amène vers le réel du borroméanisme, en imaginant ces noeuds chers à Lacan, c'est-à-dire là où des ronds se sont noués, là où "ça tient», ce que bien sûr les effets de dispersion contribuent à dénouer, les esquives, les relâchements. C'est ce que nous appellerons le point mm ou mmm (deux ou trois ronds) comme dans «les femmes m'emmerdent» (EMM p. 61), $\mathrm{m}=\mathrm{em}$ comme dans emmanuel ou emmanuscrit. Le point $\mathrm{m}$ (à multiplier $\mathrm{mm}$ ou $\mathrm{mmm}$ ) détermine un mouvement qui engendre des codes et s'ouvre à l'extension des signifiants, mère morte, mère maléfique renvoyant à un monde sans origine, modèle décentré, marque en désignant d'autres, graphème vers d'autres graphèmes, emmanuscrit, immortalité; le point $\mathrm{m}$ pourrait être le lieu de la terre sur la ligne asymptotique quand la distance du pont de la courbe à la droite tend vers zéro, mais pour s'éloigner a l'infini. Amour, humour de Meexomec avec petit ou grand $M$ dans la chaîne des noms Mauve, Marie, Membre, Machine, Mémoire. (Par exemple, la préface de Louve storée s'intitule Memorandum, avec le soustitre Mes mots rendent homme.) Le $\mathrm{m}$ renvoie au double narcissique dont 
nous avons parlé plus haut, Moi, «entre moi et moi » (LS p. 48), le redoublement du $\mathrm{m}, \mathrm{mm}=\mathrm{m}$ 'aime, «je me désire» (LS p. 22) ou sa négation «je ne m'aime pas» (LS p. 65): On connaît la fétichisation orale du mot chez Cocke, de lalangue, le narrateur-personnage de Va voir au ciel si j'y suis ne se définit-il pas aussi comme un «suceur de mots» (VVC p. 199)? Mm, hum, hum. Dans les travaux de I. Fonagy sur les bases pulsionnelles de la phonation, le «m» est sémantisé comme pulsion orale incorporante, succion de $/ \mathrm{m} /$.

Laissons circuler la lettre jusqu'à Mère, nom qu'a donné Sri Aurobindo à Mira Richard qui a organisé avec lui l'ashram d'Auroville où Cocke passera la dernière période de sa vie, assimilant leur pensée mystique $^{16}$. Thomas est le Nom-de-la-mère réelle de Cocke, de son histoire manquée, écrirait-il. Faire intervenir ici le nom de la mère réelle est simplement une façon d'impliquer une autre force $d$ 'attraction vers le point $m$ que nous pourrions supposer être le réel du noeud, «la rencontre horrifiante d'un point où s'évanouissent semblances et dissemblances, où l'Un lui-même abandonne ses pouvoirs ${ }^{17}$ ». Ainsi nous voyons qu'il est aisé d'enfermer les ronds dans une territorialité, et plus encore, dans une formation oedipienne, et cela de connivence avec le narrateur de Va voir au ciel si $j$ 'y suis : «Vers 2015 je n'étais autre qu'un petit Oedipe rêvant d'épouser sa mère perdue à jamais» (VVC p. 193). Cependant nous avons vu que le texte produit de l'inconscient, de nouveaux énoncés, d'autres désirs, et précisément le sujet affecté d'inconscient, se fait le sujet de signifiants qui forment de nouvelles chaînes. Ainsi le point $\mathrm{m}$ ne peut renvoyer qu'à d'autres points, ou se connecter avec d'autres, ou se déconnecter. Pour faire valoir cela davantage, il faudrait dresser encore d'autres cartographies, et le texte y est ouvert. Afin de déconstruire, il faut considérer que la prise d'appui du point mmm entraînant à la mémoire pour ne pas dire à la fixation, provoquera son mouvement inverse, celui de la déprise, de la défixation, du dé-sens. Ainsi, chez Cocke, le signifiant ludique, les jeux de mots contribuent à inventer ce mouvement. Pour revenir à la mère réelle que nous avons évoquée, la déprise est signifiée par le fait que la mythomanie délirante du texte est tout aussi importante que la tentation autobiographique. Par exemple, la mère morte, expression complétant le titre de l'emmanuscrit, réinscrite sous une autre forme dans la dédicace «à ma mère suicidée» est un fait autobiographique véridique. Cependant, contrairement à l'écrivain autrichien Peter Handke qui entreprend de faire le récit de la mere morte et de son suicide (bien que les circonstances soient très différentes de l'autre cas), en constituant un dossier des origines jusqu'à la mort (cf. P. Handke, le Malheur indifférent, Gallimard, Folio, 1975), Emmanuel Cocke choisit d'éviter cette anamnèse textuelle, et de détourner le texte de son titre en le faisant jouer précisément sur d'autres territoires. Autrement dit, la mère morte ne sera pas le sujet du livre, elle n'en sera que sa marque signifiante, son tatouage, sa cicatrice, sa blessure ou plus encore son imaginaire, sa métaphore structurante, ou peutêtre bien son «noyau froid». Perte narcissique de l'objet, perte d'amour, perte de sens, et instigation d'un double mouvement de la quête d'un sens 
perdu qui peut se traduire, comme chez Cocke, par une contrainte d'imaginer, une activité de jeu frénétique. En dépit de cette activité, le narrateur du texte antérieur à l'emmanuscrit s'écriait :

Je veux la Mer, la Mort, et la Mère. La Mer parce que c'est la liberté sans horizon, la Mort parce que c'est une récompense-délivrance, la Mère, parce que ça j'ai pas connu et qu'on m'a dit que ça tient chaud l'hiver. Et je vous emmerde (VVC p. 125).

Comme le montre bien André Green dans la Mère morte (1980) ${ }^{18}$, l'imago peut se constituer de toute façon dans la psyché, sans que la mère soit morte réellement. Chez Cocke, les traces du trauma MMM sont réinvesties et forment une systématique de renvoi parmi d'autres, mais dont le signifié est insaisissable comme le fantôme de Calmoya sur la mer. D'où l'ardent désir de couler dans l'irréel, dans le monde de la Dame transparente (Cf. «Tout le monde doit s'arrêter un jour» in $S F$ p. 19-20).

La quête du sens perdu permettant de rétablir la toute-puissance narcissique blessée fait du livre le support d'un fantasme d'autosuffisance grâce à la force inversante du désir : voir la série des énoncés de Dieuble s'y rattachant. Personnages réformateurs, mégalomanes, avec mission paranoïaque rénovatrice de la Décadence. «Moi, je suis envoyé sur terre pour traumatiser!» (LS p. 44). Établissement d'une mythologie manichéenne des purs et des sincères.

L'écriture, pour éviter le mélodrame, élément de survie essentiel au texte, est traversée de fuites, d'espoirs, d'échappées, d'utopie, de refuge dans les rêves futuristes, apocalyptiques. Elle veut parodier les romans de l'an 2000, et produire un simulacre de «roman électronique».

Le discours apocalyptique, comme ligne de fuite, permet de faire éclater le poids du passé, tout en se réclamant d'une vision politique du monde (contre-culturelle). C'est ici que la pose de visionnaire chez Cocke, position d'énonciation, prend toute son importance. Une «mystagogie eschatologique» ${ }^{19}$ se met en acte dans la parole, spéculation redondante sur la Fin du monde. Ainsi, le texte se joint au corpus du «genre» apocalyptique et des mystagogues de la modernité ${ }^{20}$, associés à une vision et à une exaltation mystique et cosmique. Voix d'Aurobindo en sourdine. Dans le cas qui nous occupe, il y a même surenchère de l'éloquence eschatologique, détruire le monde, être immortel, etc. On entend la voix du dernier homme, «Je suis l'un des derniers signes tracés d'eux-mêmes dans la grisaille où tout confond tout» (VCC p. 122), de celui qui prêche la fin. Perce aussi le désir de révéler une vérité, un secret mystérieux sur le début d'un autre monde. Il y a un système «d'envois» apocalyptiques : voix de la voix, Swedenborg, Unamuno. Signes de filiation au début de l'Emmanuscrit : le destinateur Gauvreau qui lui-même se réfère à Nerval (cf. son poème cité $L a$ corde où se pendait le vent EMM pp. 9-10), l'écriture se démultiplie, envoie à la mère morte destinataire. "L'envoi» apocalyptique fait sauter d'un nom à un autre, ici de manière explicite. Dans ce jeu d'envois, ici, cataclysme se 
substitue à suicide, ou à folie dans la chaîne synonymique. Énonciation de Rimbaud "- $\mathrm{j}$ 'attends de devenir un très méchant fou» (EMM p. 99) dont le poème "Vies, II" est recopié dans le roman de Cocke. La scène «d'envois» est en quelque sorte la scène d'écriture tel que le suggère Derrida, ainsi l'apocalyptique pourrait être la condition de tout discours, sans destination décidable ${ }^{21}$.

Chez Cocke, le discours apocalyptique n'est pas déconstruit, ou démystifié. Au contraire, il aboutit plutôt dans la mystification, avec parfois des relents de messianisme (cf. la nouvelle "Tout le monde doit s'arrêter un jour»). Être soi-même son propre dieu, ou nommer les multiples gourous d'une philosophie nouvelle, réels ou fictifs. Jésus Tanné se fait prêcheur et empereur d'une apothéose. Dieuble veut dieubliser totalement tout ce qui est vivant. Mais au-delà de ces héros inspirés, il semble que le texte soit dans un lieu indécidable entre le plein et le vide. D'une part le plein comme comblement du vide : obsession du phallus, réclamer un Maître ou une Maîtresse, compacité indissociable, répétition névrotique pour combler le sens, substance ultime, retour du signifié. Renoncer à la dispersion au profit de la réalité homogénéisante et distinguante des noms. (Ce sera la fonction dernière d'Auroville comme lieu d'espoir, de rassemblement institutionnel, "d'anesthésie aux coupures» selon l'expression de J.-C. Milner, d'où le désenchantement qui va s'ensuivre chez Cocke). D'autre part, l'attrait du vide, de la fuite hors-code, hors-sens, off-limits, plongée ou décollage cosmique, désir de stratosphère, d'acide, dissolutions dans la langue, retour du nouveau, le contraire de la répétition. Excéder la langue, dire en plus.

Cette oscillation nous amène pourtant, en définitive, à une pénible retombée, branchée avec ce que nous avons exposé plus haut sur le noyau froid $\mathrm{mmm}$ et la problématique narcissique, c'est-à-dire enfin à l'autocritique virulente que fait l'auteur de son écriture qu'il appelle «happening avorté» (que nous avons placée sous la rubrique de l'amphigouri par commodité et sans aucun jugement de valeur), et ceci bien sûr en rapport avec l'émergence dispersante que nous avons soulevée au début, et dont l'œuvre ne pourra pas être l'exorcisme.

C'est donc la figure (est-elle fictive?) de l'écrivain raté que nous cernons, celle du mort-né, du fœetus avorté, signe reconnaissable et itérable dans le texte de Cocke. L'amphigouri, en tant que production brouillonne, sauvage, discontinue, opère en principe la rétention de ses ratés, de ses ratages d'écriture, ratures non censurées. Corrections et épurations minimales, à quoi participent chez Cocke les nombreux jeux de mots en lapsus. Elégance et raffinement sont des valeurs bannies du système.

En rappelant certains faits déjà mentionnés, nous voulons montrer qu'Emmanuel Cocke devient prisonnier de l'image romanesque qu'il se construit lui-même entre deux narcissismes, et à partir de l'énigme du suicide maternel vécu comme traumatisme infantile engendrant des forma- 
tions textuelles singulières. Le narcissisme de $v^{22}$ se gonfle de désir mégalomane et d'autocontemplation du moi (Cf. discours de Dieuble, EMM p. 49-50 : «je vois ma trogne sur des posters géants» ou, à la fin de l'Emmanuscrit: "L'idée de repeupler l'univers à mon image, enfant par enfant, m'enchanta et $j$ 'ouvris enfin les yeux", EMM p. 236, souligné dans le texte). Être un, unique, plein comme nous disions plus haut. Surmonter la désintégration, unifier le morcellement, la dispersion, l'anarchie. Mais, nous l'avons déjà souligné, cela est vite désarticulé et joué dans le texte de Cocke, «je suis art-narcisse» (EMM p. 60) : «Et j'ai rejoint la belle au moi dormant» (VVC p. 36) et encore sur l'axe paradigmatique, "-Alors tu vas voir de quel moi je me chauffe" (VVC p. 75). L'art-narcisse ou le bois/moi ouvrent à l'altérité, c'est le deuxième aspect narcissique, l'être libidinal que nous avions relevé antérieurement chez Lacan, l'expérience du décentrement, du double androgynique, de la violence, du désespoir. Avec l'art-narcisse, la vie vient mimer la mort comme délivrance du désir. «Un plongeon vers la vie, vers la naissance» (SF p. 23). La stratification de la détresse détruit l'illisible du texte, le farfelu technique pour rendre le texte (comme on rend les armes) à sa métaphysique morbide, à ses affects de vide, à la nécropole maternelle. C'est ici qu'on peut parler de narcissisme de mort ${ }^{23}$, ce qui nous replace sur la piste de la ligne asymptotique que nous avions proposée au début, puisqu'ici, les tensions se font entre l'Un et Zéro, et qu'en l'occurrence, le point zéro dans le texte cockien touche à l'immortalité24. La douleur devient un sentiment narcissique. Le moi dépossédé et aliéné à une figure irreprésentable - du fantôme de Calmoya à la Dame transparente - fait que la maîtrise de la situation traumatique est délibérément vouée à l'échec. C'est ici que surgit avec intensité la figure mythique du raté que nous venons d'esquisser, de son défaitisme, du moins investie comme telle par le texte. «cul de sac ambulant», "faux Rimbaud raté», «faux Baudelaire raté» (LS pp. 42-43, 49, 51; SF p. 101), «microbe impuissant» (SF p. 101), "Je ne suis plus qu'un microbe butinant une micropoussière posée sur un grain de sable» (VVC p. 199), "acteur raté» (EMM p. 41), "génie et raté» (EMM p. 50), "old child raté (EMM p. 58), "ce laquet (sic) surréaliste de Dieuble» (EMM p. 124), etc. Le sujet voulant inventer un nouveau style, style total, se prend à, se regarde échouer. «Poisson noyé en ses propres eaux. Araignée s'étouffant dans ses propres fils» (SF p. 101-102). C'est ainsi que le texte de Cocke comme idéologème ${ }^{25}$ vient recouper entre autres les énoncés de Cesare Pavese dans son journal sur l'impuissance, l'amour, l'échec, l'omnipotence, la mélancolie, etc. «Il cessa de voir des sons, et le petit point orange de son impuissance alla crever comme une bulle morte à la surface du néant» (SF p. 68). Si nous voulons bien admettre cette homologie des écritures, nous pourrions dire que la thèse que soutient Dominique Fernandez à propos de Pavese est tout à fait pertinente dans le cas de Cocke, à savoir que les livres de Pavese ne le délivrent pas de ses fantômes, car il ne voulait pas en être délivré26. Nous pourrions affirmer que Cocke, comme son autre, porte dans son œuvre l'idée du suicide et "choisit l'expérience d'échec» comme pari. Dieuble proclame qu'il a choisi d'être «le roi des ratés» (EMM p. 115). Ainsi, l'écriture devient une ruse (masochiste dirait Fernandez) avec les fantasmes 
contre lesquels elle se débat. Chez Cocke, il y a cette tentation jouissive de faire de l'auto-analyse dans le roman (particulièrement dans Louve storée), faisant par conséquent rater le roman et l'auto-analyse. Avortés, ils ne peuvent être menés à terme, ni l'un ni l'autre.

À réinscrire compulsivement son angoisse «belle débauche intérieure» (VVC p. 44), «ma névrose paranoïaque» (VVC p. 7), «je suis paranoïaque» (VVC p. 181), le sujet dans son hospice mental, (se) posant avec plaisir comme un animal erratique, sanglier souffrant (il est possible de récupérer cela dans le cabotinage burlesque du texte), à réinscrire également sa mort, en tant que criminel (le) et / ou suicidaire, par exemple Serge Marcus se tuant dans l'angoisse de ne pas être publié (SF p. 103), le sujet de la parole refuse de s'en délivrer et son jeu - triste - c'est la volonté d'échec comme stratégie de langage. Re-plonger.

Pavese lui-même ne croyait pas à la valeur cathartique de l'œuvre d'art. Car le récit, au lieu de délivrer les affects en produisant son ou ses sens (le "plein des sens» écrivait Cocke), contribue à la formation des affects qu'il est censé délivrer, ils sont pris au récit. Comme le dieu Çiva de la Trinité hindoue, ça détruit et féconde. «Mais Çiva est du côté de mon ciel» (VVC p. 173). Ceci renvoie à la présence-absence du sujet, processus en jeu dans l'écriture, comme dans la cure analytique. Je ne me purge pas en racontant ${ }^{27}$. La catharsis est un effort pour rejoindre le lieu d'où l'on parle, pour réintégrer le lieu de la présence et de l'authenticité, mais comme l'a bien montré $R$. Barthes, l'écriture est aussi le départ d'un mouvement oblique qui subvertit cette quête, sape l'origine et soustrait l'œuvre à la pertinence de la vérité. C'est à la fois le lieu du sens et de sa dislocation infinie, travail acharné des esquives - je disais de la stupeur. En revanche, un problème de réception du discours, sur le plan de l'énonciation, l'impression que les effets interlocutoires du récit sont ratés ou avortés. Hantise du flop. Alun Meexomec s'écrie: «On ne m'a pas reconnu, je ne suis pas connu!!!» (LS P. 44). Le narcissisme négatif (de mort) incite au discours de l'auto-dénigrement, fantasme du déchet, du rebut qui retourne les effets interlocutoires non-accomplis contre l'énonciateur et son corps propre. «Ses mots viennent d'outre-monde via l'enfer, il sent la sueur et l'urine mal dormies, et son long corps triste encombre la chaise rassasiée, comme d'un homme qui viendrait de combler trop vite une faim vieille de mille siècles» (LS p. 41). Corps morcelé de Chunéma "divisé en plusieurs riens dispersés» (SF p. 88).

Il y a sursignification du paradigme écrire/aigrir chez Cocke, et surévaluation de l'écrivain simulacre de lui-même : "pseudo-génie infirme» (LS p. 42), névrosé russe (Brokendov), dieu-diable, ivrogne, drogué, acteur, imposteur. Peut-il y avoir en définitive, grâce à une gamme d'altérations, de fines dissociations, de changements de position, et grâce au désir du mode exaltant de la dilatation, une rupture du narcissisme? L'interrogation demeure en suspens. N'être que la trace de, l'ébauche de, le maquillage de, 
une ligne, «je n'suis plus qu'un linéament» (VVC p. 195). La ligne s'efface, se perd.

(...) il a la sensation qu'une mer immense envahit son cerveau, puis il ressent un vide intégral, très doux mais triste ${ }^{28}$.

1. Citation dans le texte original en italien : «tentazione dello scrittore. Aver scitto qualcosa che ti lascia come un fucile sparato, ancora scosso e riarso, vuotato di tutto te stesso, dove non solo hai scaricato tutto quello che sai di te stesso, ma quello che sospetti e supponi, e i sussulti, i fantasmi, l'inconscio - averlo fatto con lunga fatica e tensione, con cautela di giorni e tremori e repentine scoperte e fallimenti e irrigidirsi di tutta la vita su quel punto - accorgersi che tutto questo è come nulla se un segno umano, una parola, une presenza non lo accoglie, lo scalda - e morir di freddo - parlare al deserto - essere solo notte e giorno como un morto" (Cesare Pavese, Il mestiere di vivere, Torino, Einaudi, 1952, (27 giugno 1946), p. 289-90). L'édition française utilisée est Gallimard, Folio, Tome II, p. 415.

2. Comme l'avait fait Emmanuel de Swedenborg en 1772 (nom condensé avec celui de Borges pour former la signature Luis-Manuel Swedenborgès de la préface du texte de Cocke SexeFiction), E. Cocke avait prédit et annoncé sa mort en Inde. Dans son oeuvre, on retrouve maintes traces du corps se brisant, ceci étant lié à l'expérience du vide, de la plongée, certains énoncés à valeur prémonitoire. Ex.: «(...) j'irai m'écraser en amère mer, délivré». (Va voir au ciel si j'y suis, p. 173), ou «Est-ce d'ici qu'on va me faire plonger dans cette mer qui me fascine depuis trop longtemps, tellement que je me raccrocherai désespérément au ciel?» (Ibid., p. 200), etc. À partir du mourir par l'eau ou du fantasme suicidaire de la coulée, on pourrait brancher d'autres désirs de mort féminins, exprimés et accomplis en avancée douce cependant, sans la chute et la fracassée du corps, ceux de Virginia Woolf ou de la grande poétesse argentine Alfonsina Storni. Néanmoins, dans la diachronie affective et fraternelle de Cocke, son corps de cendres s'inscrit entre deux autres autodétruits, ceux de Claude Gauvreau et de Louis Geoffroy. Par exemple, Cocke modèle l'Emmanuscrit de la mère morte à l'écoute de la parole de l'autre, celle du poème et de la lettre de C. Gauvreau qu'il reproduit. Acte de reconnaissance de/par l'autre, satisfaction à re-voir, recopier, imprimer le fétiche manuscrit. Cela circule également chez Louis Geoffroy qui dédie son récit Emmanuelle ma nuit «à Claude Gauvreau sans qui il y aurait moins de passion, cette autre fleur baroquen et a Emmanuelle Septembre (Cf. Louis Geoffroy, Etre ange étrange, Ed. Danielle Laliberté, 1974). Rappelons que Louis Geoffroy accompagnait au saxophone Emmanuel Cocke chantant ses compositions. Il a également publié le dernier récit posthume de Cocke Sexe pour sang dans la collection qu'il dirigeait alors, «Le cadavre exquis" chez Guérin éditeur en 1974. Il y a d'évidentes affinités dans l'art de ces deux derniers, et par rapport au risque mortel qui s'y est conjoint, l'intoxication.

3. Romans : Brokendov, (1964 remanié en 1969), Québécamor (1972, écrit en Colombie, le Roman d'Auroville, en sous-titre l'Aumavatar d'Auroville (écrit à Auroville en Inde pendant l'été 1973). La nouvelle Chulalonga (1973), des pièces de théâtre, par exemple Reportage en direct chez Brandy Dynamite et Sherry Delicatessen réalisé à Radio-Canada en 1968 ou Madame Sauce-y-était, pièce jouée au Théâtre Petit Champlain à Québec en 1967. Vis en boîte paye ton air! recueil de proésimage, scénario de film : Nana et Ninif au pays des naïfs (1971), J'ai 21 siècles sous le pseudonyme d'Agnès Sorel. Manuscrits perdus de pièces radiophoniques comme la Photo du siècle (1969) ou Il ne sera pas une fois (1971) réalisées a Radio-Canada, manuscrit perdu du roman policier la Nuit de la Saint-Jean (1968). Plusieurs poèmes-chansons. Production journalistique, surtout de la critique de cinéma. Scénario et réalisation du film $35 \mathrm{~mm}$ Musika, 1965. E. Cocke était acteur dans ce film, comme dans certaines de ses pièces radiophoniques. Brokendov et Québécamor 
étaient envisagés comme scénario de films à réaliser. Soulignons que la production littéraire de Cocke s'accomplira au Québec entre 1965 et 1973, bien que les publications seront concentrées sur une période courte de deux ans, entre 1971 et 1973. (E. Cocke, né à Nantes en Bretagne le 19 mai 1945 de père américain et de mère armoricaine, choisit l'exil au Québec en décembre 1965. Malgré son engouement et son désir d'assimilation de la culture québécoise, de ses particularités linguistiques dont il injectera ses textes, il continue à se considérer comme apatride, et l'informel de sa forme littéraire en sera la démonstration).

4. E. Cocke, Va voir au ciel si j'y suis, Montréal, Ed. du Jour, 1971, p. 201. Ce roman a subi trois versions. La première, finaliste au prix du Cercle du Livre de France en 1968, s'intitulait le Ciel automatique de Monsieur Jésus Tanné, et la deuxième en 1970 s'intitulait Pour l'amour du ciel. *Pour les références textuelles à venir, nous utiliserons les abréviations suivantes : VVC pour Va voir au ciel si j'y suis, EMM pour l'Emmanuscrit de la mère morte, LS pour Louve storé, SF pour Sexe-fiction, SpS pour Sexe pour sang.

5. Le texte-rhizome est fait de dimensions, de lignes de segmentarité, de fuite, d'intensités, de vitesses d'écoulement de flux. «Un agencement est précisément cette croissance des dimensions dans une multiplicité qui change nécessairement de nature à mesure qu'elle augmente ses connexions» (G. Deleuze, F. Guattari, Rhizome, Paris, Minuit, 1976, p. 23). Il faut mentionner qu'avec les lignes de déterritorialisation, il y a circulation de particules asignifiantes également, des états de non-signes que le «corps sans organes» fait passer, et c'est simplement par rapport à cela que nous établissons une connexion arbitraire avec le réel dispersant. Car il demeure certain que par rapport a l'agencement machinique deleuzien le discours lacanien - et psychanalytique - est réfutable et en aucun cas superposable.

6. Cf. Jean-Claude Milner, les Noms indistincts, Paris, Seuil, 1983.

7. «Ressort de la fascination mortelle, alliance de l'horreur et du désir, qui trouve son accomplissement chez tels sujets pour qui le scintillement séducteur de l'objet n'est autre que sa face dispersante mème : non pas simplement ne pas supporter que la société ni le monde ni l'humanité subsistent, mais proprement désirer qu'ils ne subsistent pas, ne reconnaître pour nom d'un desir que ce qui disperserait tout nom, n'admettre pour ses demandes d'autre règle que cette dispersion même» (J.-C. Milner, Ibid., p. 67. C'est nous qui soulignons).

8. Cf. Denis Roche, Dépôts de savoir et de technique, Paris, Seuil, 1980.

9. Cf. Préface de G. Deleuze in Louis Wolfson, le Schizo et les Langues, Paris, Gallimard, 1970, pp. 5-23.

10. «Étant donné que l'origine du plaisir de l'esprit est la même - un noyau de plaisir par les mots et par le non-sens, entouré d'une coque de plaisir par levée ou par allégement d'inhibition - il s'ensuit que ces rapports analogues à l'inhibition établissent la parenté interne du naïf et du mot d'esprit» (S. Freud, le Mot d'esprit et ses Rapports avec l'inconscient, Paris, Gallimard, Idées, p. 284-285). Nous soulignons le mot «coque», cédant au plaisir homonymique créé par le mot traduit de l'allemand. L'expression «coque de plaisirn nous paraît heureuse par rapport à la levée du refoulement qu'est la jouissance de l'écriture. Faudrait-il ajouter qu'en l'occurrence, le signifiant désigne en anglais l'organe sexuel mâle. Pure coïncidence.

11. R. Barthes, les Sorties du texte, in Bataille, Colloque de Cerisy, Paris, 10/18, 1973, p. 51. (Les mots sont soulignés par l'auteur).

12. Rappelons que le Président Schreber mettait la féminité au premier plan, ayant le sentiment qu'une masse de «nerfs femelles» lui avait passé dans le corps, nerfs dont la fécondation divine immédiate engendrerait de nouveaux humains. La transformation d'homme en femme était le premier germe du système paranoïaque délirant de Schreber, ressentie d'abord comme une persécution et une injure grave. Cela se transformera par la suite en une mégalomanie mystique. Cette problématique est également une ligne de fuite du discours de Cocke qui pourrait donner lieu à une analyse sous cet angle. Cf. Daniel Paul Schreber, Mémoires d'un névropathe, Paris, Seuil, 1975, et S. Freud, Remarques 
psychanalytiques sur l'autobiographie d'un cas de paranoía, (Le Président Schreber), Paris, PUF, 1970, pp 263 à 324.

13. Cf. J. Lacan, «Les deux narcissismes» in les Écrits techniques de Freud, Séminaire livre I, Paris, Seuil, 1975, p. 144-145. Sur la question des deux narcissismes, nous reviendrons un peu plus loin sur une autre distinction, celle que fait André Green entre le narcissisme de vie et le narcissisme de mort, cela afin de mettre l'accent sur l'aspect anéantissant du second pattern narcissique mis également en évidence, mais de façon différente, par Lacan.

14. Ibid., p. 145.

15. Il en existe de multiples variations chez des écrivains aussi éloignés qu'Auguste Strindberg ou Julio Cortázar. C'est également la «thèse» soutenue dans le roman Femmes de Philippe Sollers : «Le monde appartient aux femmes. C'est-d-dire a la mort. Là-dessus, tout le monde ment» (p. 13). Voir aussi le jeu permutatif : «Le monde appartient à la mort... N'est-ce pas évident? Autant le laisser aux femmes... N'est-ce pas logique?» (p. 37). P. Sollers, Femmes, Paris, Gallimard, 1983.

16. La connaissance des écrits de Mère s'est accomplie surtout, chez Emmanuel Cocke, par la médiation des textes de Satprem, particulièrement la Genèse du surhomme.

17. “Là, et nulle part ailleurs, réside cette horreur du noeud qui s'éprouve constamment : le réel du noeud n'étant rien que l'avènement, par le dénouage borroméen, du réel comme tel (on conçoit que tels sujets s'en détournent comme de l'horreur absolue, reculant incessamment à accomplir la moindre coupure dénouante)" J.C. Milner, les Noms indistincts, Paris, Seuil, 1983, p. 14.

18. Conférence reproduite in A. Green, Narcissisme de vie narcissisme de mort, Paris, Minuit, coll. Critique, 1983, pp. 222-253.

19. Cf. J. Derrida, D'un ton apocalyptique adopté naguère en philosophie, Paris, Galilée, 1983, p. 23 (Derrida va reconsidérer le ton des «mystagogues» dénoncé par Kant comme une imposture annonçant la mort de la philosophie).

20. Derrida rapporte que selon Kant, ceux-ci «ne nous disent pas simplement qu'ils voient, touchent ou sentent. Ils pressentent, ils anticipent, ils approchent, ils flairent, ce sont les hommes de l'imminence et de la trace» Cf. Ibid., pp. 43-44.

21. Ibid., pp. $77-78$ et 95 .

22. La terminologie est empruntée à A. Green, Narcissisme de vie narcissisme de mort, Paris, Minuit, 1983.

23. A. Green définit celui-ci comme aspiration à la mort psychique. Cette tendance à l'abaissement au niveau zéro rejoint la force entropique à effet homéostatique que nous avions articulée à partir de certains textes de M.-C. Blais (cf. E. Cliche, «Un rituel de l'avidité» in Voix et Images, Vol. VIII, $\mathrm{n}^{\circ} 2$, Hiver 1983, p. 229-248).

24. «Au-delà du morcellement qui fragmente le Moi et le ramène à l'auto-érotisme, le narcissisme primaire absolu veut le repos mimétique de la mort. Il est la quête du non-désir de l'Autre, de l'inexistence, du non-être, autre forme d'accès à l'immortalité. Le Moi n'est jamais plus immortel que lorsqu'il soutient n'avoir plus d'organes, plus de corps.» A. Green, op. cit., p. 278.

25. Rappelons une définition : «Le recoupement d'une organisation textuelle (d'une pratique sémiotique) donnée avec les énoncés (séquences) qu'elle assimile dans son espace ou auxquels elle renvoie dans l'espace des textes (pratiques sémiotiques) extérieurs, sera appelé un idéologème» (J. Kristeva, «Le texte clos» in Semeiotike, Paris, Seuil, 1969, p. 114).

26. Cf. D. Fernandez, l'Echec de Pavese, Paris, Grasset, 1967. 
27. Cf. Poétique 41, Fév. 1980, pp. 2-21, sur le cas Anna O. des Etudes sur l'hystérie de Freud et Breuer : «N'est-ce pas que toute énonciation, fat-elle cathartique, produit une absence du sujet à lui-même, où s'engouffre une virtualité pressante d'être-à-dire, virtualité qui ne saurait être comblée que par un nouveau récit, lequel à son tour...» (...) «cette faille que la parole creuse dans le locuteur et qui assure la perpétuation du narratif. À présent que les mots ont commencé d'entamer la plénitude idéale de l'être, il y aura toujours à raconter, et ce n'est pas à un récit qu'on peut demander de boucler le récit du récit». L. Jenny, «Il n'y a pas de récit cathartique» in Poétique 41, p. 18.

28. E. Cocke, «Les ennemis du joualbum» in Sexe-fiction, Montreal, Éd. de l'heure, p. 46. 